EPJ Web of Conferences 47, 04001 (2013)

DOI: $10.1051 /$ epjconf/20134704001

(C) Owned by the authors, published by EDP Sciences, 2013

\title{
White dwarfs in the WTS: Eclipsing binaries
}

\author{
P.R. Steele ${ }^{1, a}$, R.P. Saglia ${ }^{1}$, J. Koppenhoefer ${ }^{2}$, M.R. Burleigh ${ }^{3}$ \\ and M. Cappetta ${ }^{1}$
}

${ }^{1}$ Max-Planck-Institut für extraterrestrische Physik, Giessenbachstrasse, 85748 Garching, Germany

${ }^{2}$ Universitäts-Sternwarte München, Scheinerstrasse 1, 81679 Munich, Germany

${ }^{3}$ Department of Physics and Astronomy, University of Leicester, University Rd., Leicester

LE1 7RH, UK

\begin{abstract}
We have identified photometric white dwarf candidates in the WFCAM transit survey through a reduced proper motion versus colour approach. Box-fitting with parameters adjusted to detect the unique signature of a white dwarf + planet/brown dwarf transit/eclipse event was performed, as well as looking for variability due to the irradiation of the companions atmosphere by the white dwarf's high UV flux. We have also performed a simple sensitivity analysis in order to assess the ability of the survey to detect companions to white dwarfs via the transit method.
\end{abstract}

\section{INTRODUCTION}

The detection and study of brown dwarf (BD) mass companions to white dwarfs (WDs) is of great importance in the study of high mass ratio binary formation and evolution. Two distinct populations are thought to be the outcome of stellar evolution; wide pairs where the secondary has migrated outwards due to the mass loss of the WDs progenitor (Farihi et al. 2006, Nordhaus et al. 2010), and the close systems in which the secondary has survived a stage of common envelope evolution and may eventually lead to the formation of a cataclysmic variable $(\mathrm{CV})$. In these close binaries, the BD is expected to be irradiated by the WD's high UV flux, leading to substantial differences in the "day" and "night" side hemispheres. Such irradiation effects can be observed at wavelengths as short as the SDSS $i^{\prime}$-band (e.g. NLTT 5306; Steele et al. 2012) but become more pronounced in the near-IR, as has been observed in WD 0137-359 (Burleigh et al. 2013, in prep.).

In this work we present an initial search in the $J$-band WFCAM Transit Survey (WTS) for closely orbiting companions to candidate WDs selected by reduced proper motion and colour cuts. We analyse the data for both transit/eclipsing events, and the effects of irradiation on the companion's atmosphere.

\section{IDENTIFYING CANDIDATE WHITE DWARFS}

Candidate WDs were selected in the WTS fields by means of reduced proper motion (RPM). Proper motions were obtained through a cross correlation with the SuperCOSMOS Sky Survey ${ }^{1}$ (SSS). An initial match within $1^{\prime \prime}$ produced a total of 49523, 49247, 53433, and 157928 objects in the $03,07,17$ and 19 hour fields respectively.

\footnotetext{
ae-mail: psteele@mpe.mpg.de

${ }^{1}$ http: //www-wfau.roe.ac.uk/sss/

This is an Open Access article distributed under the terms of the Creative Commons Attribution License 2.0, which permits unrestricted use, distribution, and reproduction in any medium, provided the original work is properly cited.
} 

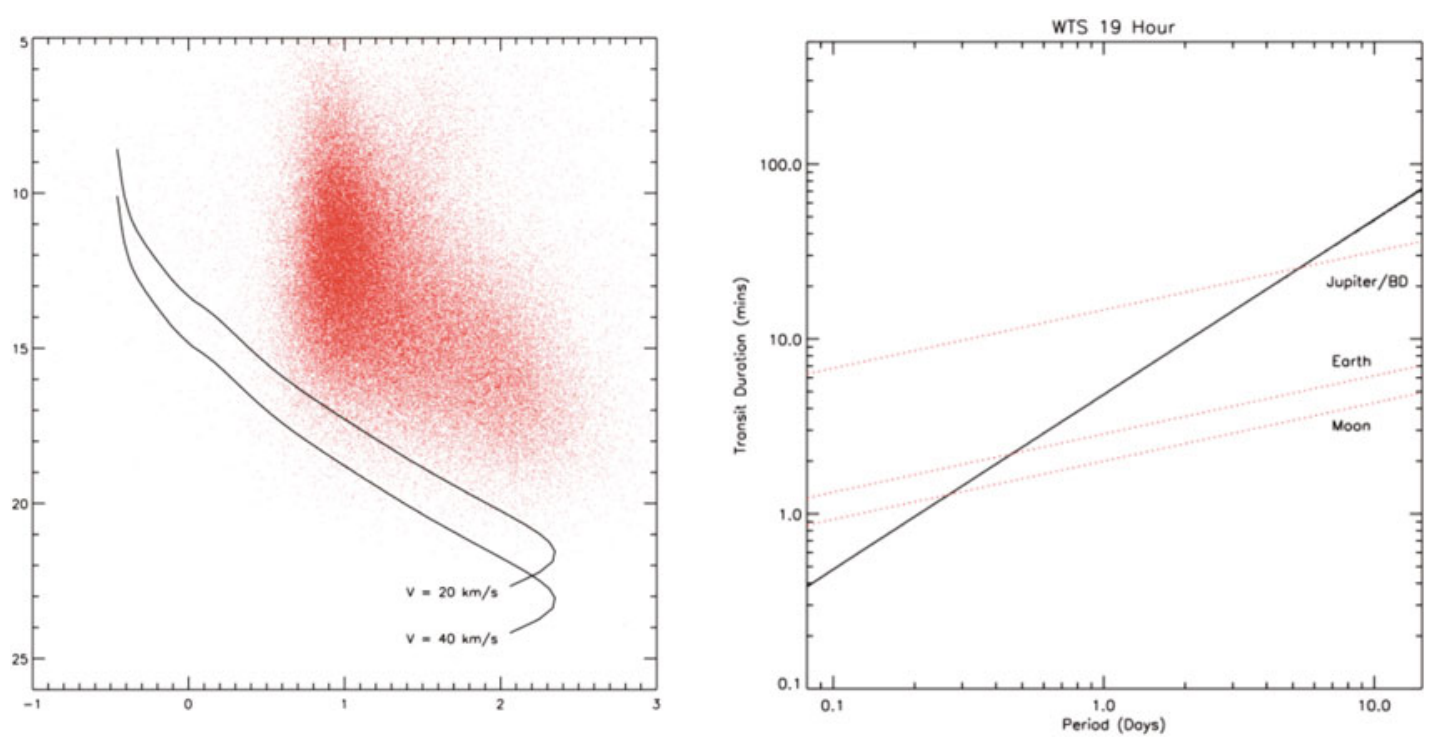

Figure 1. (a) Reduced proper motion diagram for all the stars in the WTS fields with measured proper motions from the SuperCOSMOS catalog. Over plotted are the cooling tracks for $\mathrm{H}$ (black) atmosphere WDs with $v_{\tan }=20$ and $40 \mathrm{kms}^{-1}$. (b) Limits on the ability of the WTS 19 hour field to detect eclipses or transits based on a 1 Earth radius WD. The dashed line represents the orbital period versus transit duration for an Earth sized planet (lower) and a Jupiter sized planet (upper). The 19 hour field is the most sensitive as this dataset contains the most points.

In order to select WD candidates we followed the technique of Rowell \& Hambly (2011). Out of our initial matches, 21455, 23725, 17877 and 56509 objects had full four plate detections in the 03, 07, 17 and 19 hour fields respectively. Unlike Rowell \& Hambly (2011) we are not concerned with the lower proper motion or magnitude limits of the survey. Our primary concern is to reduce the number of candidate WD light curves to a manageable number for period analysis. The unique signature of a eclipse/transit of an object around a WD will then be enough to determine if the WD is genuine. In the case of variable objects, follow-up spectroscopy would likely be needed to confirm the primary as a bona fide WD.

The reduced proper motion was calculated for each cross matched object using the following equation:

$$
H_{m}=m+5 \log _{10} \mu+5
$$

Figure 1(a) shows the RPM plotted against the colour $b_{j}-r_{59 F}$ for all objects in the WTS fields. We have over plotted the cooling tracks for DA WDs with $v_{t a n}=20 \mathrm{~km} / \mathrm{s}$ and $v_{\tan }=40 \mathrm{~km} / \mathrm{s}$. The cooling models were kindly provided by Pierre Bergeron ${ }^{2}$ in the SSS wavebands.

The cleanest sample of WD candidates would be gained by using stars positioned below the $40 \mathrm{~km} / \mathrm{s}$ line in the diagram. However, as mentioned previously the unique signature of a transit across a WD should be distinguishable from that across a sub-dwarf, the most likely contaminant of this survey. Therefore, we take all objects below the $20 \mathrm{~km} / \mathrm{s}$ line as candidate WDs, giving a total of 685, 195, 439 and 790 candidates in the $03,07,17$ and 19 hours fields respectively.

\footnotetext{
2 http://www. astro.umontreal.ca/bergeron/CoolingModels/
} 


\section{Hot Planets and Cool Stars}

Table 1. Upper limits on period in days detectable within the four WTS fields for objects ranging from Earth mass to the maximum BD mass, assuming a minimum of 5 points detected in transit/eclipse.

\begin{tabular}{ccccc}
\hline & 03 Hour & 07 Hour & 17 Hour & 19 Hour \\
\hline \hline Moon & $<0.10$ & $<0.14$ & $<0.17$ & $<0.27$ \\
Earth & $<0.17$ & $<0.24$ & $<0.27$ & $<0.47$ \\
Jupiter/BD & $<1.84$ & $<2.69$ & $<3.09$ & $<5.36$ \\
\hline
\end{tabular}

\section{SENSITIVITY}

In Faedi et al. (2010) transit times for objects orbiting WDs vary from approximately 2 minutes (a Moon sized object orbiting a $1 \mathrm{M}_{\odot}$ WD with a period of 0.22 days) to 30 minutes (a brown dwarf/gas giant orbiting with a period of 15 days). In contrast main-sequence stars host planets with transit times on the order of hours. Therefore, it may be the case that some transit surveys are not ideal in searching for these dramatically smaller transit durations.

In order to assess the sensitivity of the WTS fields to planets and BDs we performed the following simple analysis. A random sample of 100 light curves was selected from each of the four fields. Each sample was then phase folded through periods ranging from 0.08 to 15 days in steps of 0.015 days. The average time was then calculated between data points for each period, and a minimum detectable transit duration calculated assuming an absolute minimum of 5 points needed to detect a transit. The results for the 19 hour field are plotted in Fig. 1(b).

The results show that as the radius decreases, and thus the transit duration decreases we become less sensitive to larger periods. Indeed, the survey is only capable of detecting an Earth sized planet at the shortest periods, as the photometry has been folded over a much smaller time base. Increasing the size of the planet to a Jupiter/BD size increases our sensitivity to longer periods and so on with increasing radius. For the 19 hour field, which to date contains the most data points, we are sensitive to Earth detection only in the period range 0.08 to 0.5 days. The full set of results for all fields is given in Table 1.

\section{BOX-FITTING}

The box-fitting algorithm used is a modified version of that developed by Kovács et al. (2002). The modifications include 3 extra features (Zendejas et al., in prep); After the initial fit a box for a secondary transit is also fitted. This allows for use of the algorithm in detecting eclipsing binaries, a common source of contamination in such surveys. The fitted boxes are re-fitted with trapezoids in order to further account for the ingress and egress phases. The final shape therefore varies between a box and a "V", making the fit more precise. Finally, all data with a distance $<0.75 q$ (where $q$ is the fractional transit length) from the epoch $t_{0}$ are removed to mask the signal and the algorithm repeated. The results give a $S / N$ of the light curve with the transit removed. A very low $S / N-S / N_{\text {removed }}$ is a good discriminator between planet candidates and binaries.

The computation time of the box-fitting algorithm can be significantly reduced if we only apply it to a physically meaningful range. As can be seen in Table 1 we are unlikely to be able to detect a transiting planet around a WD in the WTS with a period greater than 6 days. The Roche limit, at which a planet would be pulled apart by tidal forces, is given by Faedi et al. (2011) as 0.08 days. The eclipse duration of a planet around a WD is roughly $\frac{1}{60}$ of the orbital period. Therefore, we explore a period range of 0.08 to 6 days using $1,000,001$ steps, with a transit duration of 0.001 to 0.1 phase units. The data points are re-sampled in the folded light curves into 500 bins. We extract the 6 best fitting periods from each star.

Following the box-fitting analysis, we chose the folded light curve for each star with the lowest $\chi^{2}$ value out of six. We then eliminated any light curve with alias detections close to periods of $0.33,0.5$, 

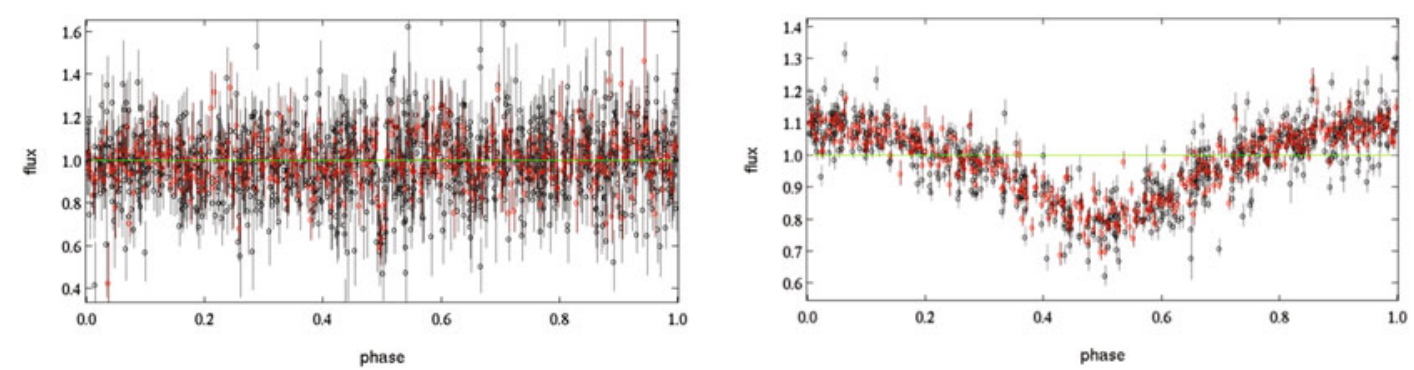

Figure 2. Example candidates from the box-fitting analysis of WDs in the WTS. Left: a tentative transit candidate in the 19 hour field. The short transit duration of only 5 mins makes this star ideal for follow-up photometry. Right: a variable candidate. In this case we may be viewing the irradiation effect of the WD's flux on a brown dwarf's atmosphere.

0.66, 1.0 and 2.0 days. Finally we ordered the final light curves in decreasing order of $S / N$ in order to place the best potential candidates at the top of the viewing list. Each light curve was then assessed by eye to search for credible candidates. Example candidates can be seen in Fig. 2.

\section{SUMMARY}

We have selected WD candidates in the WTS via a reduced proper motion versus colour approach. The candidates were analysed for transit/eclipse events as well as variability due to irradiation effects in the companion's atmosphere using a modified box-fitting algorithm. Candidates for follow-up were selected using transit duration as a key indicator as to whether the star is a possible bona fide WD. Variable candidates were also selected for potential follow-up. A simple sensitivity analysis suggests that the WTS is indeed sensitive to transit/eclipse events around white dwarfs, but only for increasingly small periods as the radius of the companion decreases.

\section{References}

[1] Faedi, F., West, R. G., Burleigh, M. R., Goad, M. R. \& Hebb, L., MNRAS 410, 899 (2011)

[2] Farihi, J., Hoard, D. W., \& Wachter, S., ApJ 646, 480 (2006)

[3] Kovacs, G., Zucker, S. \& Mazeh, T., A\&A 391, 369 (2002)

[4] Nordhaus, J., Spiegel, D. S., Ibgui, L., Goodman, J. \& Burrows, A., MNRAS 408, 631 (2010)

[5] Rowell, N. \& Hambly, N. C., MNRAS 417, 93 (2011)

[6] Steele, P. R., Saglia, R. P., Burleigh, M. R., Marsh, T. R., Gansicke, B. T., Lawrie, K., Cappetta, M., Girven, J. \& Napiwotzki, R., MNRAS arxiv1212:2899 (2012)

[7] Zendejas, J., Koppenhoefer, J. and Saglia, R.P., in prep. 